\title{
EXPLICIT REPRESENTATION OF SUPER-MINIMAL $J$-HOLOMORPHIC CURVES IN A 6-DIMENSIONAL SPHERE
}

\author{
Hideya HaSHIMOTO
}

\begin{abstract}
We give the differential equation of first order with respect to a superminimal $J$-holomorphic curves of $S^{6}$ and write down the holomorphic horizontal curve in the complex quadric corresponds to the Boruvka sphere in $S^{6}$.
\end{abstract}

\section{Introduction}

Let $S^{6}$ be a 6 -dimensional unit sphere centered at the origin in the purely imaginary octonians $\operatorname{Im} \boldsymbol{O}$ which is identified with a 7-dimensional Euclidean space. It is known that there exists the canonical nearly Kähler structure on $S^{6}$ which is not integrable. Let $M^{2}$ be a Riemann surface. We call a immersion from $M^{2}$ into $S^{6}$ a $J$-holomorphic curve of $S^{6}$ if each tangent space of $M^{2}$ is invariant under the almost complex structure. In ([Br1]), R. L. Bryant has constructed super-minimal $J$-holomorphic curves of $S^{6}$ as follows ;

Let $G_{2}$ be the exceptional simple Lie group of automorphisms of the octonions. It is known that the homogeneous space $G_{2} / U(2)$ coincide with the Grassmannian manifold $G_{2}(\operatorname{Im} \boldsymbol{O})$ of oriented 2-planes in $\operatorname{Im} \boldsymbol{O}$. Then we have the $\boldsymbol{C P} \boldsymbol{P}^{2}$-bundle over $S^{6}$ such that $\pi: G_{2} / U(2)\left(=G_{2}(\operatorname{Im} \boldsymbol{O})\right) \rightarrow G_{2} / S U(3)\left(=S^{6}\right)$. Let $H(q)$ be the horizontal subspace of $G_{2}(\operatorname{Im} \boldsymbol{O})$ at $q \in G_{2}(\operatorname{Im} \boldsymbol{O})$ with respect to the $S U(3)$ connection of $S^{6}$. Let $L_{+}(q)$ be the subspace (of $H(q)$ ) of the vectors on which $\pi_{*}$ is complex linear with respect to the canonical complex structure of $G_{2}(\operatorname{Im} O)$ and the nearly Kähler structure on $S^{6}$. Then it can be shown that the dimension of the space $L_{+}(q)$ is four and $\cup_{q \in G_{2}(\operatorname{Im} O)} L_{+}(q)$ is the subbundle of rank 4 of the tangent bundle of $G_{2}(\operatorname{Im} \boldsymbol{O})$. Let $\phi: M^{2} \rightarrow G_{2}(\operatorname{Im} \boldsymbol{O})$ be a holomorphic map from a Riemann surface $M^{2}$ to the Grassmaniann manifold $G_{2}(\operatorname{Im} \boldsymbol{O})$ whose differential image $d \psi\left(T_{p} M^{2}\right)$ is included in $L_{+}(p)$ for any point $p \in M$. Then we have the superminimal $J$-holomorphic curve $\pi \circ \phi: M^{2} \rightarrow S^{6}$, locally.

This construction is to solve the first order differential equations by twistor

Mathematical Subject Classifications (1991): 53B25, 53C15

Key words : nearly Kähler structure, super-minimal $J$-holomorphic curves

Received February 28, 1997. 
and moving frame methods. In this paper, we give another proof of the R. L. Bryant's construction by purely algebraic direct calculation. As an application, we write down the holomorphic horizontal curve with respect to the Boruvka sphere $S^{2}(1 / 6)$ to $S^{6}(1)$, explicitly. Our method is essentially same as that R.L. Bryant's one, but its idea is based on S.S. Chern's method ([Ch]). We use the same notational convention to the previous paper ([H1]).

The author wishes to express his sincere thanks to Professor K. Sekigawa for his many valuable suggestions, discussions and encouragement.

\section{Preliminaries}

Let $M^{2}$ be a $J$-holomorphic curves in a 6-dimensional sphere $S^{6}$, then it is a minimal surface in $S^{6}$. It is known that there exists the following fibre bundle structure;

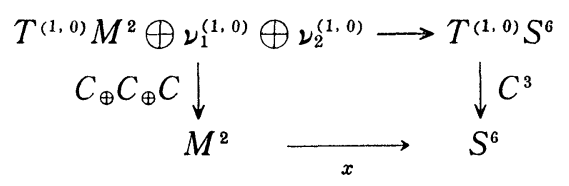

where $T^{(1,0)} M^{2}$ is a complexified tangent bundle of type $(1,0), \nu^{(1,0)}$ is a complexified 1st normal bundle of type $(1,0)$ and $\nu_{2}^{(1,0)}$ is a complexified 2 nd normal bundle of type $(1,0)$ (for details in ([B2] or [H1])). Let $\left\{U_{\alpha}\right\}_{\alpha \in A}$ be a sufficiently fine finite covering of $M^{2}$. Let $\left(f_{3}, f_{2}, f_{1}\right)$ be a local section of the bundle $T^{(1,0)} M^{2} \oplus \nu_{1}^{(1,0)} \oplus \nu_{2}^{(1,0)}$ on $U_{\alpha}$, and $\left(f_{3}^{\prime}, f_{2}^{\prime}, f_{1}^{\prime}\right)$ another section on $U_{\beta}$. We assume that $U_{\alpha} \cap U_{\beta} \neq \emptyset$, then we have

$$
\left(f_{3}^{\prime}, f_{2}^{\prime}, f_{1}^{\prime}\right)=\left(f_{3}, f_{2}, f_{1}\right)\left(\begin{array}{ccc}
e^{i \theta} & 0 & 0 \\
0 & e^{2 i \theta} & 0 \\
0 & 0 & e^{-3 i \theta}
\end{array}\right),
$$

where $\theta$ is a real valued function on $U_{\alpha} \cap U_{\beta}$. By (2.1), we can define a map from $M^{2}$ to a (complex) 5-dimensional quardrics $Q^{5} \subset \boldsymbol{P}^{6}(\boldsymbol{C})$ such that

$$
\left[f_{1}\right]: M^{2} \longrightarrow Q^{5}
$$

where [ ] denotes the homogeneous coordinate of $\boldsymbol{P}^{6}(\boldsymbol{C})$. From the above relations of the transition functions, this map is well defined on whole on $M^{2}$

Remark. Here $f_{1}$ is considered as a $C^{\urcorner} \backslash\{0\}$ valued function; $f_{1}: U_{\alpha} \rightarrow C^{7} \backslash\{0\}$.

Unfortunately, the map $\left[f_{1}\right]$ is not holomorphic map, but we can derive a holomorphic data from $\left[f_{1}\right]$. Next we give the fundamental relations of the immersion $x: M^{2} \rightarrow S^{6}$ and $\left[f_{1}\right]$. Since the octonians is a non-commutative normed algebra, there exists the exterior product $u \times v=(1 / 2)(\bar{v} u-\bar{u} v)$ for any 
$u, v \in O$. Let $\boldsymbol{C} \otimes_{\boldsymbol{R}} \boldsymbol{O}$ be the complexification of the octonions $\boldsymbol{O}$. We extend the exterior product complex linearly on $\boldsymbol{C} \otimes_{\boldsymbol{R}} \boldsymbol{O}$ and denote the same symbol. Then we see that

$$
2 \sqrt{-1} f_{1} \times \bar{f}_{1}=x .
$$

In fact, since $f_{1}=(1 / 2)(a-\sqrt{-1} a \times x)$, for some $a \in \operatorname{Im} O,|a|^{2}=1$, we get

$$
\begin{aligned}
& 2 \sqrt{-1} f_{1} \times \bar{f}_{1}=2 \sqrt{-1}\left(\frac{1}{2}(a-\sqrt{-1} a \times x) \times \frac{1}{2}(a+\sqrt{-1} a \times x)\right) \\
& =a \times(a \times x)=-a^{2} x=x .
\end{aligned}
$$

\section{Fundamental properties of super-minimal $J$-holomorphic curves}

First, we derive the holomorphic data from $f_{1}$. Let $U$ be a sufficiently small open subset of $M^{2}$. Then $f_{1}$ can be considered as a vector valued function on $U, f_{1}: U \rightarrow \boldsymbol{C}^{\eta} \backslash\{0\}$, which satisfy $\left\langle f_{1}, f_{1}\right\rangle=0$ and $\left\langle f_{1}, \bar{f}_{1}\right\rangle=1 / 2$. Taking account of structure equations of the simple Lie group $G_{2}$, (Weingarten formula $(5.8)$ in $[\mathrm{H} 1]$, or $(2.16)$ in $[\mathrm{Br} 1])$, we have

$$
d f_{1}=f_{1} \kappa_{1}{ }^{2}+f_{2} \kappa_{1}{ }^{2}+f_{3} \kappa_{1}{ }^{3}-\bar{f}_{2} \theta^{3} .
$$

Since we take the special unitary frame $\left\{f_{1}, f_{2}, f_{3}\right\}$, we have $\kappa_{1}{ }^{3} \equiv 0$. Now we assume a $J$-holomorphic curve is superminimal; $\kappa_{1}{ }^{2} \equiv 0$. By (3.1), we have

$$
d f_{1}=f_{1} \kappa_{1}{ }^{1}-\bar{f}_{2} \theta^{3},
$$

where $\theta^{3} \in \Lambda^{(1,0)}(U)$. By (3.2), we have

$$
\frac{\partial f_{1}}{\partial \bar{z}}=f_{1} \kappa_{1}{ }^{1}\left(\frac{\partial}{\partial \bar{z}}\right)
$$

If we put $\xi=\gamma \cdot f_{1}$ such that $\gamma: U \rightarrow C$ is a complex valued function on $U$. By (3.3), we have

$$
\begin{aligned}
\frac{\partial \xi}{\partial \bar{z}} & =\frac{\partial \gamma}{\partial \bar{z}} \cdot f_{1}+\gamma \cdot \frac{\partial f_{1}}{\partial \bar{z}} \\
& =f_{1}\left(\frac{\partial \gamma}{\partial \bar{z}}+\gamma \cdot \kappa_{1}{ }^{1}\left(\frac{\partial}{\partial \bar{z}}\right)\right) .
\end{aligned}
$$

From this we see that $\xi: U \rightarrow C^{\eta} /\{0\}$ is the holomorphic map if and only if $\gamma$ is a solution of the following differential equation;

$$
\frac{\partial \gamma}{\partial \bar{z}}+\gamma \cdot \kappa_{1}{ }^{1}\left(\frac{\partial}{\partial \bar{z}}\right)=0
$$

In ([G-H: p. 5]), by $\bar{\partial}$-Poincaré Lemma in one variable, we can solve the differential equation (3.4). By using this solution, we can get a holomorphic map $\xi: U \rightarrow C^{7} \backslash\{0\}$ locally. Since $f_{1}$ satisfies $\left\langle f_{1}, f_{1}\right\rangle=0$, by direct calculation, we 
get $\langle\xi, \xi\rangle=0$ and $\xi \neq 0$. The above argument we get the following

Proposition 3.1. Let $x: M^{2} \rightarrow S^{6}$ be a super minimal J-holomorphic curve of $S^{6}$. Then the map $\xi: M^{2} \rightarrow Q^{5}$ is a holomorphic map.

Next, our purpose is to prove the converse of Proposition 3.1. We shall prove the following

THEOREM 3.2. Let $\xi: M^{2} \rightarrow C^{7} \backslash\{0\} \cong \boldsymbol{C} \otimes_{\boldsymbol{R}} \operatorname{Im} \boldsymbol{O} \backslash\{0\}$ is a holomorphic map satisfying the following conditions;

$$
\begin{gathered}
\langle\xi, \xi\rangle=0, \\
\frac{d \xi}{d z} \neq 0, \\
\frac{d \xi}{d z} \times \bar{\xi} \neq 0, \\
\left\langle\frac{d \xi}{d z}, \xi \times \bar{\xi}\right\rangle=0 .
\end{gathered}
$$

Then the map

$$
\frac{\sqrt{-1 \xi} \times \bar{\xi}}{|\xi|^{2}}: M^{2} \longrightarrow S^{6}
$$

is a super minimal J-holomorphic curve of $S^{6}$.

Remark. The condition (1) is equivalent that $\xi$ is contained in a 5-dimensional complex quadric. The condition (2) is equivalent that $\xi$ is an immersion. The condition (3) means that the $\operatorname{map}(\sqrt{-1} \xi \times \bar{\xi}) /|\xi|^{2}: M^{2} \rightarrow S^{6}$ is an immersion. The condition (4) means that the $\operatorname{map}(\sqrt{-1 \xi} \times \bar{\xi}) /|\xi|^{2}$ is a $J$-holomorphic curve in $S^{6}$. In particular, the condition (4) is important to construct the immersion.

In order to prove Theorem 3.2, we prepare some facts. We assume that $\xi$ satisfy the conditions (1)-(4). We may show that the condition (4) is equivalent to the immersion $x$ is a superminimal $J$-holomorphic curve in $S^{6}$. If we put

$$
\frac{\xi}{|\xi|}=\alpha+\sqrt{-1} \beta
$$

where $\alpha$ and $\beta$ are $\operatorname{Im} \boldsymbol{O}$-valued functions, and $|\xi|=\sqrt{\langle\xi, \bar{\xi}\rangle}$. Then we have

$$
0=\left\langle\frac{\xi}{|\xi|}, \frac{\xi}{|\xi|}\right\rangle=\langle\alpha, \alpha\rangle-\langle\beta, \beta\rangle+2 \sqrt{-1}\langle\alpha, \beta\rangle
$$

and

$$
1=\left\langle\frac{\xi}{|\xi|}, \frac{\bar{\xi}}{|\xi|}\right\rangle=\langle\alpha, \alpha\rangle+\langle\beta, \beta\rangle
$$


Hence we have

$$
\langle\alpha, \alpha\rangle=\langle\beta, \beta\rangle=\frac{1}{2},
$$

and

$$
\langle\alpha, \beta\rangle=0 \text {. }
$$

We need two identities to calculate exterior product of $\operatorname{Im} \boldsymbol{O}$ as follows;

Moufang identity

$$
(u v)(w u)=u(v w) u
$$

for any $u, v, w \in \boldsymbol{O}$

The identity of exterior product

$$
\begin{gathered}
u \times v=u v+\langle u, v\rangle 1 \\
(u \times v) \times v=\langle u, v\rangle v-\langle v, v\rangle u,
\end{gathered}
$$

for any $u, v \in \operatorname{Im} \boldsymbol{O}$.

Also we need the following two lemmas

LEMMA 3.3. The map $\xi: M^{2} \rightarrow Q^{5}$ is holomorphic if and only if

$$
\begin{aligned}
& \frac{1}{2}\left(\frac{\partial \alpha}{\partial x}-\frac{\partial \beta}{\partial y}\right)=\mu \alpha-\nu \beta \\
& \frac{1}{2}\left(\frac{\partial \alpha}{\partial y}+\frac{\partial \beta}{\partial x}\right)=\nu \alpha+\mu \beta
\end{aligned}
$$

where $\mu$ and $\nu$ are some real valued functions on an open subset $U\left(\subset M^{2}\right)$ and $z=$ $x+\sqrt{-1} y$ is an isothermal coordinates on $U$.

Proof of Lemma 3.3. Since $\xi$ is a holomorphic, we have

$$
\frac{\partial}{\partial \bar{z}}\left(\frac{\xi}{|\xi|}\right)=\frac{\partial}{\partial \bar{z}}\left(\frac{1}{|\xi|}\right) \xi \text {. }
$$

If we put $\mu+\sqrt{-1} \nu=(\partial / \partial \bar{z})(1 /|\xi|)$, then we get

$$
\text { L.H.S. of }(3.10)=\frac{1}{2}\left(\frac{\partial \alpha}{\partial x}-\frac{\partial \beta}{\partial y}\right)+\sqrt{-1}\left(\frac{\partial \alpha}{\partial y}+\frac{\partial \beta}{\partial x}\right) \text {. }
$$

On the other hand, we have

$$
\text { R.H.S. of }(3.10)=\mu \alpha-\nu \beta+\sqrt{-1}(\nu \alpha+\mu \beta) \text {. }
$$

So we get the desired result.

LEMMA 3.4. Let $\alpha, \beta$ be functions defined by (3.5), then we have 


$$
\begin{aligned}
& \left(\frac{\partial \alpha}{\partial x} \times \beta\right) \times(\alpha \times \beta)=\frac{1}{2} \alpha \times \frac{\partial \beta}{\partial y}+2\left\langle\frac{\partial \beta}{\partial y}, \alpha \times \beta\right\rangle \beta \\
& \left(\alpha \times \frac{\partial \beta}{\partial x}\right) \times(\alpha \times \beta)=\frac{1}{2} \frac{\partial \alpha}{\partial y} \times \beta+2\left\langle\frac{\partial \alpha}{\partial y}, \alpha \times \beta\right\rangle \beta \\
& \left(\frac{\partial \alpha}{\partial y} \times \beta\right) \times(\alpha \times \beta)=-\frac{1}{2} \alpha \times \frac{\partial \beta}{\partial x}-2\left\langle\frac{\partial \beta}{\partial x}, \alpha \times \beta\right\rangle \beta \\
& \left(\alpha \times \frac{\partial \beta}{\partial y}\right) \times(\alpha \times \beta)=-\frac{1}{2} \frac{\partial \alpha}{\partial y} \times \beta-2\left\langle\frac{\partial \alpha}{\partial x}, \alpha \times \beta\right\rangle \beta
\end{aligned}
$$

Proof of Lemma 3.4. By Moufang identity and (3.6)-(3.9), we have

L.H.S. of $(3.11)=-\left(\beta \times \frac{\partial \alpha}{\partial x}\right) \times(\alpha \times \beta)$

$$
\begin{aligned}
& =-\left(\left\langle\beta, \frac{\partial \alpha}{\partial x}\right\rangle 1+\beta \cdot \frac{\partial \alpha}{\partial x}\right) \times(\alpha \cdot \beta) \\
& =-\left\langle\beta, \frac{\partial \alpha}{\partial x}\right\rangle \alpha \cdot \beta-\left\langle\beta \cdot \frac{\partial \alpha}{\partial x}, \alpha \cdot \beta\right\rangle-\left(\beta \cdot \frac{\partial \alpha}{\partial x}\right) \cdot(\alpha \cdot \beta) .
\end{aligned}
$$

By (3.6), we get

$$
\left\langle\beta \cdot \frac{\partial \alpha}{\partial x}, \alpha \cdot \beta\right\rangle=0
$$

By Moufang identity, we have

$$
\text { L.H.S. of }(3.11)=-\left\langle\beta, \frac{\partial \alpha}{\partial x}\right\rangle \alpha \cdot \beta-\beta \cdot\left(\frac{\partial \alpha}{\partial x} \cdot \alpha\right) \cdot \beta \text {. }
$$

By (3.6) and (3.8), we get

$$
\left\langle\beta, \frac{\partial \alpha}{\partial x}\right\rangle=-\nu
$$

Also, by (3.8), we have

$$
\frac{\partial \alpha}{\partial x} \cdot \alpha=\frac{\partial \beta}{\partial y} \cdot \alpha-\mu-2 \nu \beta \alpha .
$$

By (3.15) and (3.16), we get

$$
\text { L.H.S. of }(3.11)=-\beta\left(\frac{\partial \beta}{\partial y} \cdot \alpha\right) \beta-\frac{1}{2} \mu \text {. }
$$

Now we calculate $\beta((\partial \beta / \partial y) \cdot \alpha) \beta$. By (3.6), we get

$$
\begin{aligned}
\beta\left(\frac{\partial \beta}{\partial y} \cdot \alpha\right) \beta & =\beta\left(\frac{\partial \beta}{\partial y} \cdot \alpha-2\left\langle\frac{\partial \beta}{\partial y} \cdot \alpha, \beta\right\rangle \beta+2\left\langle\frac{\partial \beta}{\partial y} \cdot \alpha, \beta\right\rangle \beta\right) \beta \\
& =\beta\left(\frac{\partial \beta}{\partial y} \cdot \alpha-2\left\langle\frac{\partial \beta}{\partial y} \cdot \alpha, \beta\right\rangle\right) \beta+2\left\langle\frac{\partial \beta}{\partial y} \cdot \alpha, \beta\right\rangle \beta^{3} .
\end{aligned}
$$


Since we have

$$
\left\langle\frac{\partial \beta}{\partial y} \cdot \alpha-2\left\langle\frac{\partial \beta}{\partial y} \cdot \alpha, \beta\right\rangle \beta, \beta\right\rangle=0, \quad \text { and } \quad \beta^{3}=-\frac{1}{2} \beta .
$$

Hence we have

$$
\begin{aligned}
\beta\left(\frac{\partial \beta}{\partial y} \cdot \alpha\right) \beta & =-\beta\left(\left(\frac{\partial \beta}{\partial y} \cdot \alpha-2\left\langle\frac{\partial \beta}{\partial y} \cdot \alpha, \beta\right\rangle \beta\right) \bar{\beta}\right)-\left\langle\frac{\partial \beta}{\partial y} \cdot \alpha, \beta\right\rangle \beta \\
& =\beta^{2}\left(\frac{\partial \beta}{\partial y} \cdot \alpha-2\left\langle\frac{\partial \beta}{\partial y} \cdot \alpha, \beta\right\rangle \beta\right)-\left\langle\frac{\partial \beta}{\partial y}, \alpha \times \beta\right\rangle \beta \\
& =-\frac{1}{2}\left(\bar{\alpha} \cdot \frac{\bar{\partial} \beta}{\partial y}-2\left\langle\frac{\partial \beta}{\partial y} \cdot \alpha, \beta\right\rangle \bar{\beta}\right)-\left\langle\frac{\partial \beta}{\partial y}, \alpha \times \beta\right\rangle \beta \\
& =-\frac{1}{2} \alpha \cdot \frac{\partial \beta}{\partial y}-2\left\langle\frac{\partial \beta}{\partial y}, \alpha \times \beta\right\rangle \beta \\
& \left.=-\frac{1}{2} \mu-\frac{1}{\alpha} \alpha \times \frac{\partial \beta}{\partial y}-2<\frac{\partial \beta}{\partial y}, \alpha \times \beta\right\rangle \beta
\end{aligned}
$$

By (3.17) and (3.18), we have (3.15). Similarly we get (3.12)-(3.14).

Now we are in a position to prove Theorem 3.2 .

Proof of Theorem 3.2. We put the map $\tilde{x}=(\sqrt{-1} \xi \times \bar{\xi}) /|\xi|^{2}$. We can easily see that

$$
\tilde{x}=2 \alpha \times \beta .
$$

Also, by direct calculations, we get

$$
d \tilde{x}\left(\begin{array}{c}
\partial \\
-\partial \bar{z}
\end{array}\right)=\frac{\sqrt{-1}}{|\xi|^{2}} \frac{d \xi}{d z} \times \bar{\xi}+\frac{d}{d z}\left(\frac{1}{|\xi|^{2}}\right) \sqrt{-1} \xi \times \bar{\xi} .
$$

Hence, if $|\xi|^{2} \neq 0$ and $(d \xi / d z) \times \bar{\xi} \neq 0$ then $x$ is an immersion from $M^{2}$ to $S^{6}$. Next we may show that the following identity under the conditions (1) to (4) are satisfied:

$$
d \tilde{x}\left(\begin{array}{c}
\frac{\partial}{\partial z} \\
-\frac{1}{2}
\end{array}\right) \times \sqrt{-1} d \tilde{x}\left(\frac{\partial}{\partial z}\right)
$$

on $M^{2}$. In fact, by (3.19), we get

$$
\begin{aligned}
\text { L.H.S. of }(3.20) & =d \tilde{x}\left(\frac{\partial}{\partial z}\right) \times \tilde{x}=4 \frac{\partial(\alpha \times \beta)}{\partial z} \times(\alpha \times \beta) \\
& =2 \frac{\partial(\alpha \times \beta)}{\partial x} \times(\alpha \times \beta)-2 \sqrt{-1} \frac{\partial(\alpha \times \beta)}{\partial y} \times(\alpha \times \beta) .
\end{aligned}
$$

On the other hand, we have 


$$
\text { R.H.S. of }(3.20)=\sqrt{-1} d \tilde{x}\left(\frac{\partial}{\partial z}\right)=\sqrt{-1} \frac{\partial(\alpha \times \beta)}{\partial x}+\frac{\partial(\alpha \times \beta)}{\partial y} \text {. }
$$

Therefore the identity (3.20) is satisfied if and only if

$$
\begin{aligned}
& \frac{\partial(\alpha \times \beta)}{\partial x} \times(\alpha \times \beta)=\frac{1}{2} \frac{\partial(\alpha \times \beta)}{\partial y}, \\
& \frac{\partial(\alpha \times \beta)}{\partial y} \times(\alpha \times \beta)=-\frac{1}{2} \frac{\partial(\alpha \times \beta)}{2 \partial x} .
\end{aligned}
$$

Therefore, by Lemma 3.4, the identities (3.21) and (3.22) are holds, if and only if

$$
\left\langle\frac{\partial \beta}{\partial y}, \alpha \times \beta\right\rangle=\left\langle\frac{\partial \alpha}{\partial y}, \alpha \times \beta\right\rangle=\left\langle\frac{\partial \beta}{\partial x}, \alpha \times \beta\right\rangle=\left\langle\frac{\partial \alpha}{\partial x}, \alpha \times \beta\right\rangle=0
$$

From this and (3.5) the identities (3.21) and (3.22) are satisfied if and only if the condition (4) holds. We get the desired result.

\section{Holomorphic horizontal curves in $G_{2}(\operatorname{Im} O)$}

The purpose of this section is to prove the following Theorem 4.1, by applying Theorem 3.2. We set a basis of $\boldsymbol{C} \otimes_{\boldsymbol{R}} \operatorname{Im} \boldsymbol{O}$ by $\varepsilon=(0,1) \in \boldsymbol{H} \oplus \boldsymbol{H}, E_{1}=i N$, $E_{2}=j N, E_{3}=-k N, \bar{E}_{1}=i \bar{N}, \bar{E}_{2}=j \bar{N}$ and $\bar{E}_{3}=-k \bar{N}$, where $N=(1-\sqrt{-1 \varepsilon}) / 2, \bar{N}=$ $(1+\sqrt{-1} \varepsilon) / 2 \in \boldsymbol{C} \otimes_{\boldsymbol{R}} \boldsymbol{O}$ and $\{1, i, j, k\}$ is the canonical basis of $\boldsymbol{H}$. Then we have $\left\langle E_{\imath}, E_{\jmath}\right\rangle=\left\langle\bar{E}_{\imath}, \bar{E}_{\jmath}\right\rangle=\langle N, N\rangle=\langle\bar{N}, \bar{N}\rangle=0,\left\langle E_{\imath}, \bar{E}_{\jmath}\right\rangle=(1 / 2) \delta_{i \jmath}$, and $\langle N, \bar{N}\rangle=$ $1 / 2$.

THEOREM 4.1. Let $\xi: C \rightarrow Q^{5}$ be the holomorphic map defined as follows;

$$
\begin{aligned}
\xi= & E, 2+\bar{E}_{2}(2 \sqrt{6} z)+\bar{E}_{3}\left(2 \sqrt{-1} \sqrt{15} z^{2}\right)+\varepsilon\left(2 \sqrt{ } 10 z^{3}\right) \\
& +E_{3}\left(2 \sqrt{-1} \sqrt{15} z^{4}\right)+E_{2}\left(2 \sqrt{6} z^{5}\right)+\bar{E}_{1}\left(-2 z^{6}\right),
\end{aligned}
$$

where $\left\{\varepsilon, E_{1}, E_{2}, E_{3}, \bar{E}_{1}, \bar{E}_{2}, \bar{E}_{3}\right\}$ is the basis of $\boldsymbol{C} \otimes_{\boldsymbol{R}} \operatorname{Im} \boldsymbol{O}$ (defined above). Then the map

$$
\frac{\sqrt{-1 \xi \times \bar{\xi}}}{|\xi|^{2}}: P^{1}(C) \longrightarrow S^{6}
$$

coincide with the Boruvka sphere (superminimal $J$-holomorphic curve of $S^{6}$ ) $\phi_{0}$ : $S^{2}(1 / 6) \rightarrow S^{6}(1)$.

Proof of Theorem 4.1. We may show that $\xi$ satisfies 4-conditions in Theorem 3.2. By direct calculation, we get

$$
\langle\xi, \xi\rangle=2\left(-2 z^{6}\right)+(2 \sqrt{6} z)\left(2 \sqrt{6} z^{5}\right)-\left(2 \sqrt{15} z^{2}\right)\left(2 \sqrt{15} z^{4}\right)+\left(2 \sqrt{10} z^{3}\right)^{2}=0
$$

So we have $\xi: C \rightarrow Q^{5}$. Hence the condition (1) of Theorem 3.2 is satisfied. 
Since the $\xi$ is a polynomial of order 6 , we may check that the corresponding infinitesimal conditions at $z=0$. We have

$$
\begin{gathered}
\xi(0)=2 E_{1}, \\
\sqrt{-1} \xi(0) \times \overline{\xi(0)}=4 \sqrt{-1} E_{1} \times \bar{E}_{1}=2 \varepsilon, \\
\xi^{\prime}(0)=\bar{E}_{2}(2 \sqrt{6}) .
\end{gathered}
$$

Hence we have

$$
\left\langle\xi^{\prime}(0), \xi(0) \times \overline{\xi(0)}\right\rangle=\left\langle\bar{E}_{2}(2 \sqrt{6}),-2 \sqrt{-1} \varepsilon\right\rangle=0 .
$$

Also we have

$$
\begin{aligned}
& \xi^{\prime \prime}(0)=\bar{E}_{3}(4 \sqrt{-1} \sqrt{15}), \quad\left\langle\xi^{\prime \prime}(0),-2 \sqrt{-1} \varepsilon\right\rangle=0 . \\
& \xi^{\prime \prime \prime}(0)=\varepsilon(12 \sqrt{10}), \\
& \left\langle\xi^{\prime \prime \prime}(0),-2 \sqrt{-1 \varepsilon\rangle}+\left\langle\xi^{\prime \prime}(0), \xi^{\prime}(0) \times \overline{\xi(0)}\right\rangle\right. \\
& =\langle\varepsilon(12 \sqrt{10}),-2 \sqrt{-1} \varepsilon\rangle \\
& \quad+\left\langle\bar{E}_{3}(4 \sqrt{-1} \sqrt{15}), \bar{E}_{2}(2 \sqrt{6}) \times 2 \bar{E}_{1}\right\rangle=0 . \\
& \left\langle\xi^{(4)}(0), \xi(0) \times \overline{\xi(0)}\right\rangle+2\left\langle\xi^{\prime \prime \prime}(0), \xi^{\prime}(0) \times \overline{\xi(0)}\right\rangle=0, \\
& \left\langle\xi^{(5)}(0), \xi(0) \times \overline{\xi(0)}\right\rangle+3\left\langle\xi^{(4)}(0), \xi^{\prime}(0) \times \overline{\xi(0)}\right\rangle \\
& \quad+2\left\langle\xi^{\prime \prime \prime}(0), \xi^{\prime \prime}(0) \times \overline{\xi(0)}\right\rangle=0, \\
& \left\langle\xi^{(6)}(0), \xi(0) \times \overline{\xi(0)}\right\rangle+4\left\langle\xi^{(5)}(0), \xi^{\prime}(0) \times \overline{\xi(0)}\right\rangle \\
& \quad+5\left\langle\xi^{(4)}(0), \xi^{\prime \prime}(0) \times \overline{\xi(0)}\right\rangle=0 .
\end{aligned}
$$

Hence at $z=0, \xi$ satisfy condition (4). Next we shall calculate $\xi \times \bar{\xi}$, by using the following table of the exterior product of $\boldsymbol{C} \otimes_{\boldsymbol{R}} \boldsymbol{O}$. (See the next page.)

We can easily see that $\bar{\xi}$ is given by;

$$
\begin{aligned}
\bar{\xi}= & N\left(2 \sqrt{-1} \sqrt{10} \bar{z}^{3}\right)+\bar{N}\left(-2 \sqrt{-1} \sqrt{10} \bar{z}^{3}\right) \\
& +E_{1}\left(-2 \bar{z}^{3}\right)+\bar{E}_{1} 2 \\
& +E_{2}(2 \sqrt{6} \bar{z})+\bar{E}_{2}\left(2 \sqrt{6} \bar{z}^{5}\right) \\
& +E_{3}\left(-2 \sqrt{-}-1 \sqrt{15} \bar{z}^{2}\right)+\bar{E}_{3}\left(-2 \sqrt{-1} \sqrt{ } 15 \bar{z}^{4}\right) .
\end{aligned}
$$




\begin{tabular}{|c|c|c|c|c|c|c|c|c|}
\hline columm $\times$ row & $N$ & $E_{1}$ & $E_{2}$ & $E_{3}$ & $\bar{N}$ & $\bar{E}_{1}$ & $\bar{E}_{2}$ & $\bar{E}_{3}$ \\
\hline$N$ & 0 & $-E_{1}$ & $-E_{2}$ & $-E_{3}$ & $-\frac{\sqrt{-1}}{2} \varepsilon$ & 0 & 0 & 0 \\
\hline$E_{1}$ & $E_{1}$ & 0 & $-\bar{E}_{3}$ & $\bar{E}_{2}$ & 0 & $-\frac{\sqrt{-1}}{2} \varepsilon$ & 0 & 0 \\
\hline$E_{2}$ & $E_{2}$ & $\bar{E}_{3}$ & 0 & $-\bar{E}_{1}$ & 0 & 0 & $-\frac{\sqrt{-1}}{2} \varepsilon$ & 0 \\
\hline$E_{3}$ & $E_{3}$ & $-\bar{E}_{2}$ & $\bar{E}_{1}$ & 0 & 0 & 0 & 0 & $-\frac{\sqrt{-1}}{2} \varepsilon$ \\
\hline $\bar{N}$ & $\frac{\sqrt{-1}}{2} \varepsilon$ & 0 & 0 & 0 & 0 & $-\bar{E}_{1}$ & $-\bar{E}_{2}$ & $-\bar{E}_{3}$ \\
\hline $\bar{E}_{1}$ & 0 & $\frac{\sqrt{-1}}{2} \varepsilon$ & 0 & 0 & $\bar{E}_{1}$ & 0 & $-E_{3}$ & $E_{2}$ \\
\hline $\bar{E}_{2}$ & 0 & 0 & $\frac{\sqrt{-1}}{2} \varepsilon$ & 0 & $\bar{E}_{2}$ & $E_{3}$ & 0 & $-E_{1}$ \\
\hline $\bar{E}_{3}$ & 0 & 0 & 0 & $\frac{\sqrt{-1}}{2} \varepsilon$ & $\bar{E}_{3}$ & $-E_{2}$ & $E_{1}$ & 0 \\
\hline
\end{tabular}

Hence we get

$$
\begin{aligned}
\sqrt{-1 \xi \times \bar{\xi}=} & i(-2 \sqrt{10})\left(z^{3}+\bar{z}^{3}\right)\left(1+|z|^{2}\right)^{3} \\
& +j(2 \sqrt{15})\left(z^{2}+\bar{z}^{2}\right)\left(1+2|z|^{2}-2|z|^{6}-|z|^{8}\right) \\
& +k(-2 \sqrt{6} \sqrt{-1}(z-\bar{z}))\left(1-5|z|^{4}-5|z|^{6}+|z|^{10}\right) \\
& +\varepsilon 2\left(1-6|z|^{2}-15|z|^{4}+15|z|^{8}+6|z|^{10}-|z|^{12}\right) \\
& +i \varepsilon(-2 \sqrt{10})\left(\sqrt{-1}\left(z^{3}-\bar{z}^{3}\right)\right)\left(1+|z|^{2}\right)^{3} \\
& +j \varepsilon(2 \sqrt{15})\left(\sqrt{-1}\left(z^{2}-\bar{z}^{2}\right)\right)\left(1+2|z|^{2}-2|z|^{6}-|z|^{8}\right) \\
& +k \varepsilon(-2 \sqrt{6}(z+\bar{z}))\left(1-5|z|^{4}-5|z|^{6}+|z|^{10}\right) .
\end{aligned}
$$

Next we show that the $\operatorname{map}(\sqrt{-1 \xi \times \bar{\xi}}) /|\xi|^{2}$ coincide with the Boruvka sphere. To prove this, we take a coordinate as follows;

$$
y_{1}=\frac{|z|^{2}-1}{|z|^{2}+1}, \quad y_{2}+\sqrt{-1} y_{3}=\frac{2 z}{|z|^{2}+1} .
$$

Then we have $y_{1}{ }^{2}+y_{2}{ }^{2}+y_{3}{ }^{2}=1$. By (4.2), we get

$$
\begin{aligned}
\sqrt{-1}-1 \xi \times \bar{\xi}= & \frac{2^{5}}{(1-y)^{6}}\left\{i(-\sqrt{10}) y_{2}\left(y_{2}{ }^{2}-3 y_{3}{ }^{2}\right)\right. \\
& +j(-2 \sqrt{15}) y_{1}\left(y_{2}{ }^{2}-y_{3}{ }^{2}\right)
\end{aligned}
$$




$$
\begin{aligned}
& +k(\sqrt{6}) y_{3}\left(y_{2}{ }^{2}+y_{3}{ }^{2}-4 y_{1}{ }^{2}\right) \\
& +\varepsilon 2 y_{1}\left(3 y_{2}{ }^{2}+3 y_{3}{ }^{2}-2 y_{1}{ }^{2}\right) \\
& +i \varepsilon(\sqrt{10}) y_{2}\left(3 y_{2}{ }^{2}-y_{3}{ }^{2}\right) \\
& \left.+j \varepsilon(4 \sqrt{15}) y_{1} y_{2} y_{3}\right) \\
& \left.+k \varepsilon(\sqrt{6}) y_{2}\left(y_{2}{ }^{2}+y_{3}{ }^{2}-4 y_{1}{ }^{2}\right)\right\} \\
& =\frac{2^{5}}{(1-y)^{6}} \phi_{1} .
\end{aligned}
$$

Then we have $\left\langle\phi_{1}, \phi_{1}\right\rangle=16$ so we get

$$
\frac{\sqrt{-1} \xi \times \bar{\xi}}{|\xi|^{2}}=\frac{1}{4} \psi_{1}
$$

From this representation, we obtain the $\operatorname{map}(1 / 4) \phi_{1}: S^{2}(1) \rightarrow S^{6}(1)$ which is not isometric immersion. By direct calculation, the Gauss curvature of the induced metric of $(1 / 4) \phi_{1}$ is identically $1 / 6$. We get the desired result.

Remark. More precisely, $S^{2}$ is simply connected and Theorem 9.3 in [H1], we get the desired conclusion by standard monodromy argument.

\section{REFERENCES}

[Br1] R.L. BRYANT, Submanifolds and special structures on the octonians, J. Differential Geom., 17 (1982), 185-232.

[Br2] R.L. BRYANT, Minimal surfaces of constant curvature in $S^{n}$, Trans. Amer. Math. Soc., 290 (1985), 259-271.

[Ch] S.S. CHERN, On minimal spheres in the four spheres, Selected papers, Springer Verlag, New York, 1978, 421-434.

[G-H] G. Griffiths AND J. HarRis, Principles of algebraic geometry, Wiley-Interscience, New York, 1978.

[H-L] R. Harvey and H.B. Lawson, Calibrated geometries, Acta Math., 148 (1982), 47-157.

[H-S] H. Hashimoto and K. Sekigawa, Minimal surfaces in a 4-dimensional sphere, Houston J. Math., 21 (1995), 449-464.

[H1] H. HASHIMOTO, J-holomorphic curves in a 6-dimensional sphere (to appear).

[Se] K. SEKIgAwa, Almost complex submanifolds of a 6-dimensioanl sphere, Kodai Math. J., 6 (1983), 174-185.

[Sp] M. SPIVAK, A Comprehensive Introduction to Differential Geometry IV, Publish or Perish., 1975.

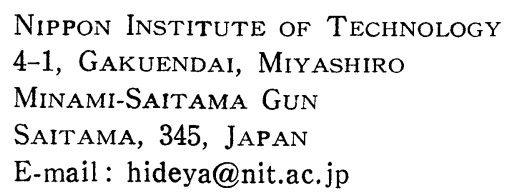

